\title{
Calidad de vida y sustentabilidad: una nueva
}

ciudadanía*

\section{Quality of Life and Sustainability: A New Citizenship}

\section{Qualité de vie soutenable : vers une nouvelle citoyenneté}

\section{Pedro César Cantú-Martínez**}

Recibido: 2015-01-20 // Aprobado: 2015-03-15 // Disponible en linea: 2015-06-30

Como citar este artículo: Cantú-Martínez, P. C. (2015). Calidad de vida y sustentabilidad: una nueva ciudadanía. Ambiente y Desarrollo, 19(37), 09-21. http://dx.doi.org/10.11144/ Javeriana.ayd19-37.cvsn

doi:10.11144/Javeriana.ayd19-37.cvsn

\section{Resumen}

El presente artículo hace una revisión sobre el concepto de calidad de vida y su dependencia del desarrollo sustentable, términos que se han constituido en centro de atención y reflexión en nuestra sociedad. A partir de ello se retoma la configuración social y colectiva, en la cual convergen los trazos de los conceptos de calidad de vida y desarrollo sustentable, que permiten acoger y justificar la figura social de una nueva construcción teórica denominada "ciudadanía del mundo frugal y segura", que se distingue por su carácter global y sustentable, y además tiene una fisonomía propia, la cual responde a las exigencias de su contexto actual, al conjugar los criterios básicos de equidad social, armonía ecológica y eficiencia económica.

Palabras clave: calidad de vida; sustentabilidad; ciudadanía; frugalidad; seguridad

* En este artículo se revisan desde una perspectiva analítica los resultados de investigaciones sobre la calidad de vida y el desarrollo sustentable, con el fin de dar cuenta de los avances y las tendencias de desarrollo en esta materia.

** Ph.D. en Ciencias Biológicas. Profesor e investigador de la Universidad Autónoma de Nuevo León, México, Facultad de Ciencias Biológicas. San Nicolás de los Garza, N.L., México. Correos electrónicos: cantup@hotmail.com; pedro.cantum@uanl.mx. 


\begin{abstract}
This article reviews the quality of life concept and how it depends on sustainable development. Both terms have turned into a relevant focus of attention and reflection in our society. Based on this, we retake the social and collective configuration, where the path of the concepts of quality of life and sustainable development overlap. This allows us to take up and justify the social figure of a new theoretical construct called "frugal and safe citizenship of the world", which is set apart by its global and sustainable character. Also, it has a particular set of characteristics as a response to the demands of its current context, by including the basic criteria of social equity, ecological harmony, and economic efficiency.
\end{abstract}

Keywords: quality of life; sustainability; citizenship; frugality; safety

\title{
Résumé
}

Le présent article fait une révision du concept de qualité de vie et sa dépendance du développement soutenable, ces conceptions sont au centre de la réflexion d'une manière importante dans notre société. A partir de cela on prend le façonnement social et collectif, dans lequel découlent les traits des concepts de qualité de vie et développement soutenable, que permettent d'accueillir et justifier la figure sociale d'une nouvelle construction théorique appelée « citoyenneté du monde frugal et assuréı qu'on distingue par son caractère global et soutenable, en plus cette figure a une physionomie propre, laquelle répond aux exigences de son contexte actuel, dans la conjugaison des critères basiques d'équité sociale, harmonie écologique et efficacité économique.

Mots-clés: qualité de vie; soutenable; citoyenneté; frugalité; assuré 


\section{Introducción}

En las últimas décadas se ha reconocido que vivimos en una complejidad social muy diversa e indeterminada, donde además los términos no son bastante claros; por lo tanto, se incrementan las complicaciones, con la inserción de nuevos contenidos, como por ejemplo la calidad de vida y el desarrollo sustentable (Flórez, 2002; Cantú-Martínez, 2012, 2013a). Estos conceptos, en los inicios del siglo xxi, ocupan un lugar importante en el marco de la política internacional, concretamente, en el contexto de las políticas socioeconómicas y ambientales (Cantú-Martínez, 2013b).

Calidad de vida es un término que suele ser empleado en muy diversas situaciones. Es usual su aplicación por líderes, diplomáticos, gobernantes, economistas, profesionales de la salud y sociólogos, entre otros, pues se trata de un vocablo que puede ser estudiado por distintas disciplinas y que está relacionado con aspectos de bienestar humano y satisfacción, producto de la observación y vivencia de los propios actores (García-Viniegras, 2005). Por otra parte, suele llamarse desarrollo sustentable al "desarrollo que satisface las necesidades del presente sin comprometer las capacidades que tienen las futuras generaciones para satisfacer sus propias necesidades", de acuerdo con la definición de la Comisión Mundial para el Medio Ambiente y el Desarrollo (1988, p. 67), que posteriormente fue enmendada en Johannesburgo en el año 2002, al añadirse que se persigue además la eliminación de la pobreza y consolidar la equidad social, tanto intergeneracionalmente como intrageneracionalmente (Cantú-Martínez, 2008; Unión Internacional para la Conservación de la Naturalez [UicN], 2008). El desarrollo, que se cimienta en el empleo del capital humano y en la explotación de los recursos naturales - con un enfoque fragmentado en las cuestiones económicas, sociales y ambientales - por si solo como proceso, se constituyó en una actividad que hace crecer las brechas socioeconómicas y ambientales entre las colectividades. Así, por una parte, persisten sociedades caracterizadas por la baja producción y la miseria, y por otra parte, en otras hay un nivel mucho más alto de consumo, riqueza y bienestar material.

Elizalde Hevia (2003) sostiene que la génesis de este problema reside en el principio del crecimiento económico permanente e ilimitado, que por mucho tiempo fue considerado la vía principal para acceder al desarrollo. Este principio se caracterizaba por disparidad en el bienestar social de las personas, relegando los aspectos de calidad ambiental y descuidando la instauración de oportunidades para las minorías. En este sentido, Ferrer (2010, p. 8) asevera que "en la actualidad, el sistema mundial enfrenta problemas no resueltos. En primer lugar, las asimetrías en los niveles de bienestar derivadas de la desigualdad en la distribución de los frutos del progreso técnico entre los países y dentro de cada uno de ellos".

La problemática en mención ha representado para la sociedad internacional eludir el escenario que conlleva dos eventualidades de carácter moral, como son las altas tasas de crecimiento poblacional y la falta de justicia social. El problema demográfico se constituye en el eje conductor del proceso de cambio global, esencialmente por el incremento en el número de personas, pero además por la manera como interactuamos con la naturaleza, especialmente para satisfacer nuestras necesidades (Xercavins, Cayuela, Cervantes y Sabater, 2005), mientras que la falta de justicia social se evidencia en la carencia de equidad e integración social. Los aspectos antes citados son fundamentales para el desarrollo humano sustentable y para acceder a una calidad de vida digna (Filmus, 1996; Sierra Fonseca, 2001).

En este escrito entendemos el desarrollo sustentable, particularmente fuerte, como la vía para mejorar sostenidamente y de forma equitativa la calidad de vida del ser humano, edificado este desarrollo sustentable a partir de pautas apropiadas de protección, preservación y conservación del ambiente, con una explotación razonable de los recursos endógenos, de forma tal que se garanticen las expectativas y los intereses de las generaciones futuras, como también los de las actuales. Así, partimos del planteamiento de que los conceptos de calidad de vida y desarrollo sustentable confluyen en la construcción necesaria de una nueva ciudadanía, que implica una dinámica como conciencia de lo local, regional y mundial. Por lo tanto, abordamos los aspectos de la definición de calidad de vida y 
sus dimensiones, para posteriormente observar la concordancia de los conceptos de calidad de vida y desarrollo sustentable que confluyen en una postura teorizante de un nuevo tipo de ciudadanía. Finalmente, hacemos algunas consideraciones de cierre.

\section{¿Qué es la calidad de vida?}

La noción de calidad de vida, según Barranco (2009), procede del año 1932 y se le adjudica su uso inicial al economista Pigou, pero su aplicación más generalizada fue a partir del comienzo de la década de los sesenta. De acuerdo con Urzúa y Caqueo-Urizar (2012, p. 62), el empleo de la noción de calidad de vida "puede remontarse a los Estados Unidos después de la Segunda Guerra Mundial, como una tentativa de los investigadores de la época de conocer la percepción de las personas acerca de si tenían una buena vida o si se sentían financieramente seguras". Sin embargo, es un hecho irrefutable que el concepto de calidad de vida ha sido utilizado de manera indistinta; por una parte, se han hecho aproximaciones al evaluar aspectos como la satisfacción con la vida, el bienestar subjetivo, la cuantificación de bienes materiales, el estado de salud-enfermedad y las condiciones de vida, entre muchas otras. Esta gran multiplicidad de conceptos puede proceder de la complejidad de la expresión, o bien de que se trata de un enunciado de carácter multidisciplinario. En la tabla 1 se presentan algunas definiciones provenientes de fuentes primarias y secundarias.

La calidad de vida, como se puede ver en las definiciones de la tabla 1, es un constructo bastante complejo que ha conllevado un extenso debate entre los académicos en el mundo, particularmente para su valoración, debido a los diferentes componentes que la pueden integrar y a la diversidad de factores que la determinan, ya que maneja escalas tanto cuantitativas como cualitativas (Robaina, 2011). Como señalan Salas Zapata y Grisales Romero (2010, p. 9): "Aun cuando la calidad de vida puede estar determinada por factores objetivos, su manifestación esencial se aprecia en el orden subjetivo, reflejo de una relación dialéctica entre lo objetivo y lo subjetivo". En otras palabras, la calidad de vida objetiva y la percibida son dos agregados de elementos que interactúan permanentemente en el individuo, y su interpretación depende de los valores que subsisten y los eventos que se suscitan en su entorno (Arita Watanabe, 2005; Nava Galán, 2012).

\section{Dimensiones de la calidad de vida}

Precisar la calidad de vida es y ha sido una labor ardua, que ha quedado inconclusa, aunque la han retomado académicos e investigadores que durante muchos años han realizado grandes intentos por precisarla; sin embargo, no se ha logrado avanzar hacia un verdadero consenso acerca de las dimensiones objetivas y subjetivas que deberían considerarse para este fin.

Tradicionalmente, en los colectivos sociales, la determinación de la calidad de vida se ha centrado en métodos de carácter objetivo, sustentados en la elaboración de medidas, tales como tasar los bienes materiales y de servicios con que cuenta la población. No obstante, a pesar de las serias dificultades que plantea el concepto de calidad de vida, López Vizcaíno y Sánchez Fernández (2009) advierten que existen tres premisas que considerar para su evaluación: la primera de ellas concierne a la satisfacción reconocida por el individuo, la segunda a las condiciones de vida en que subsiste la persona, y la tercera a una experiencia percibida de las dos anteriores.

En otras palabras, la calidad de vida de cualquier conglomerado social está ligada a su grado de desarrollo, pero además requiere el marco de referencia de otros grupos sociales que seguir y con los cuales se coteja. En este orden de ideas, es necesario precisar que el grado de desarrollo logrado por una sociedad obedece a indicadores de carácter socioeconómico que detallan el estado general de la economía de una nación, pero además debe contemplar las relaciones de orden social que guardan y prevalecen en la existencia concreta de las personas (Carpio, Pacheco, Flores y Canales, 2000). 
Ardila (2003) sostiene que las principales dimensiones de carácter objetivo que se deberían tomar en cuenta para el estudio de la calidad de vida deben referirse a los siguientes siete aspectos: bienestar emocional; riqueza material y bienestar material; salud; trabajo y otras formas de actividad productiva; relaciones familiares y sociales; seguridad; e integración con la comunidad. Por otra parte, sería importante considerar aspectos subjetivos relacionados con las personas en los factores de intimidad, expresión emocional, seguridad percibida, productividad personal y salud percibida. Además, desde un enfoque constructivista y sistémico-ecológico, como señala Barranco (2009), la calidad de vida se va edificando socialmente y colectivamente, e intervienen los propios individuos, considerando los distintos escenarios de orden personal, familiar, institucional y comunitario que se constituyen en ámbitos espacio-temporales en los cuales las personas pasan de microambientes a macroambientes. Por lo tanto, la calidad de vida, según Torres Tovar (2010, p. 8), es "plural y no singular, en el sentido que la cualificación de la sensación de existir la crea el individuo con sus múltiples determinaciones generadas por su historia ontogénica”.

Tabla 1.

Enunciaciones de calidad de vida

\begin{tabular}{|c|c|}
\hline Enunciación & Referencia \\
\hline $\begin{array}{l}\text { "el concepto calidad de vida [...] se asocia globalmente con las } \\
\text { condiciones materiales en que se desenvuelve la existencia de los } \\
\text { individuos y, más en concreto, con la experiencia personal que resulta } \\
\text { de dichas condiciones". }\end{array}$ & $\begin{array}{l}\text { Castańeda (citado en } \\
\text { Rodríguez y García } \\
(2005 \text {, pp. } 51-52)\end{array}$ \\
\hline $\begin{array}{l}\text { "calidad de vida es simplemente la suma de todas las cosas que la } \\
\text { gente consume colectivamente ya sea a través del gasto público o sea } \\
\text { porque no son comparables de ninguna manera". }\end{array}$ & $\begin{array}{l}\text { Jacobs (1996, pp. } \\
393-394)\end{array}$ \\
\hline $\begin{array}{l}\text { "considera la calidad de vida como un agregado de componentes } \\
\text { objetivos y subjetivos. Cada componente incluye siete aspectos: } \\
\text { bienestar material, salud, productividad, intimidad, seguridad, lugar } \\
\text { en la comunidad y bienestar emocional". }\end{array}$ & $\begin{array}{l}\text { Cummins (1997), } \\
\text { Lora }(2008, \text { p. } 10)\end{array}$ \\
\hline $\begin{array}{l}\text { "La calidad de vida es el resultado de la compleja interacción } \\
\text { entre factores objetivos y subjetivos; los primeros constituyen } \\
\text { las condiciones externas: económicas, sociopolíticas, culturales } \\
\text { y ambientales que facilitan o entorpecen el pleno desarrollo del } \\
\text { hombre, de su personalidad. Los segundos están determinados por la } \\
\text { valoración que el sujeto hace de su propia vida". }\end{array}$ & $\begin{array}{l}\text { Cabrera, Agostini, } \\
\text { López y Victoria } \\
\text { (citados en García- } \\
\text { Viniegras, 2005, p. 7) }\end{array}$ \\
\hline $\begin{array}{l}\text { "como un conjunto de cosas deseables no siempre reconocidas por } \\
\text { el mercado, que, al igual que la sostenibilidad, tiene dimensiones } \\
\text { económicas, sociales y medioambientales. Abarca aspectos como } \\
\text { la renta, las condiciones laborales y de alojamiento, la salud y la } \\
\text { educación, las relaciones de raza y género, la seguridad, las condiciones } \\
\text { medioambientales, las oportunidades de esparcimiento, cosas que en } \\
\text { su conjunto pueden verse como el estándar de vida general". }\end{array}$ & $\begin{array}{l}\text { Johnston (citado en } \\
\text { Sánchez de Madariaga, } \\
\text { 2004, p. 102) }\end{array}$ \\
\hline $\begin{array}{l}\text { "Calidad de vida es un estado de satisfacción general, derivado de } \\
\text { la realización de las potencialidades de la persona. Posee aspectos } \\
\text { subjetivos y aspectos objetivos. Es una sensación subjetiva de } \\
\text { bienestar físico, psicológico y social. Incluye como aspectos subjetivos } \\
\text { la intimidad, la expresión emocional, la seguridad percibida, la } \\
\text { productividad personal y la salud objetiva. Como aspectos objetivos } \\
\text { el bienestar material, las relaciones armónicas con el ambiente físico y } \\
\text { social y con la comunidad, y la salud objetivamente percibida". }\end{array}$ & Ardila (2003, p. 163) \\
\hline
\end{tabular}

Fuente: elaboración propia con base en las referencias citadas 
Sin embargo, se debe considerar, como señala Bustelo Graffigna (2008), que la subjetividad, aunque se construye autónomamente, puede ser modificada por las acciones, a través de las políticas públicas o de poder ejercidas sobre las personas. El ejemplo más ilustrativo es la forma de transculturización contemporánea que se suscita en las colectividades como producto de la globalización, donde es evidente cómo lo medios de comunicación masivos intervienen en la construcción del concepto de calidad de vida, donde esta suele ser evaluada a partir de los modelos de las sociedades más empoderadas económicamente (Macía Arce, 2009).

Hoy en día, las alusiones referentes a la calidad de vida se relacionan estrechamente con el desarrollo, esto es, el proceso mediante el cual se aspira a mejorar las condiciones económicas y sociales de la población (Cantú-Martínez, 2012). Bajo el enfoque actual de desarrollo humano, este se sustenta primordialmente en el planteamiento de Amartya Sen, que lo designa como desarrollo con libertad, en donde lo más relevante "no es que las personas tengan más cosas, sino que sean más capaces, es decir, que sean más libres y puedan vivir una vida más larga, eludir enfermedades evitables, tener acceso a la reserva de conocimientos, entre otras tantas posibilidades valiosas" (Hernández, 2008, p. 32); sin embargo, esto todavía no logra materializarse.

\section{Ciudadanía del mundo frugal y segura: convergencia de la calidad de vida y la sustentabilidad}

De acuerdo con Guevara (2010), la noción de calidad de vida surge como constructo social en la década de los setenta, producto de los vertiginosos cambios de orden social que se daban, particularmente, en el marco de las sociedades industriales que transitaban a la post-industrialización. En esta transición social, el apogeo económico y técnico-científico proveyó la apertura al empleo intensivo, extensivo y absurdo de los recursos naturales, y también se privilegió el avance de la tecnología por otros recursos como los de carácter cultural, humanos e institucionales, buscando con apremio el crecimiento económico, para la acumulación de capital financiero y material, exclusivamente (Cantú-Martínez, 2012). Este crecimiento económico, convertido en un objetivo en sí mismo, demanda en un nuevo orden social, la subordinación de aspectos tan esenciales en lo colectivo como la actividad científica y financiera, la formación profesional y la de la misma vida social, mientras que en el plano conductual de las personas, ello involucra los sistemas educativos y los medios masivos de comunicación, soslayando los factores culturales y de idiosincrasia de las personas (Herrera et al., 2004).

Esto creó una preocupación social de orden universal, que fue patente por primera vez en Estocolmo en 1972 y después conllevó tres reuniones más: Río de Janeiro en 1992, Johannesburgo en el 2002, y nuevamente Río de Janeiro, en el 2012, la cual se le llamó "Río +20". Las declaraciones emanadas de estos encuentros dejan en su contenido claramente, como lo patentiza Rull (2010, p. 106), que "el capital reproducible y el natural son directamente interdependientes, de forma que cualquier incremento en el primero termina, a la corta o a la larga, por diezmar el segundo, bien sea en forma de reducción, de contaminación o de acumulación de deshechos". Por otra parte, uno de los fines trazados en estas promulgaciones fue el dejar de manera evidente que es necesario gestionar una condición de vida con dignidad, en el marco de los derechos universales de todos los seres humanos, con el propósito de liberarla de conductas inciviles en las múltiples dimensiones en que se desenvuelve (ver tabla 2).

En estos encuentros internacionales se han examinado las causas y, desde puntos de vista de carácter ético y jurídico, se han evaluado las consecuencias funestas sobre el capital natural, el sistema ambiental global y el contexto socioeconómico en el cual subiste el ser humano. Esto ha promovido grandes debates, que se siguen dando todavía, por la forma en que las estructuras sociales hacen uso de los recursos naturales y bienes materiales (Cantú-Martínez, 2013c). Debe recordarse que si partimos del inicio de la Revolución Industrial en 1760 (Southcliffe Ashton, 2008), ya son más de 250 años de un proceso de indolencia socioambiental, en el cual las transformaciones tecnológicas e industriales han suscitado un desarrollo continuo cuyos logros son notables, pero acompañados no obstante de 
manifestaciones como la contaminación, el deterioro ambiental y la exclusión social del poder, el conocimiento y el bienestar, en colectividades vulnerables como los campesinos, los indígenas y los pobres (Cantú-Martínez, 2012). También se ha observado el poder que ostentan los medios de comunicación masivos, sujetos sin embargo a intereses de grupos minoritarios (Sierra Fonseca, 2001).

De esta manera, el concepto de calidad de vida está estrechamente enlazado a la sustentabilidad, y su preponderancia es tal que es imposible abordar la calidad de vida sin reflexionar a la vez acerca de los objetivos planteados por el desarrollo sustentable. La definición de desarrollo sustentable es un oxímoron, que contempla el desarrollo con una condición de durabilidad y en evolución, la cual favorece tanto el desarrollo del ser humano como al entorno que lo rodea. Este entorno permite además aumentar las alternativas con las que cuentan las personas para acceder a mejores niveles de bienestar, en función de aspectos tan variados como "las libertades políticas, económicas y sociales, hasta las posibilidades ofrecidas a cada uno de estar en buena salud, instruido, productivo, creativo, y de vivir en la dignidad y el pleno gozo de los derechos del hombre" (Hernández-Vela Salgado, 2001, p. 81). En este sentido, nos aproximamos al constructo social de la calidad de vida de todo ser humano. En el marco de una moralidad social de mínimos, ella debe garantizar y responder a los intereses y a las necesidades de convivencia que deben existir en toda sociedad, con acuerdos que deben trascender al orden internacional (Peña Guerrero, 2001), ya que las personas hoy en día "no solo se sienten miembros de una comunidad en particular, sino también de una comunidad internacional de cuyos problemas no pueden abstraerse".

En la anterior aseveración convergen los trazos de los conceptos de calidad de vida y desarrollo sustentable, con el fin de examinar las relaciones y prácticas sociales entre las naciones, las colectividades y las personas, donde los copartícipes de estas relaciones, ya sea en lo grupal, o bien en lo individual, afronten la exigencia de acordar y adecuar su conducta, actividades y decisiones, a pautas, principios y valores que se juzguen como los más apropiados socialmente, de acuerdo con el periodo histórico y la prospectiva que se quiera vivir; esto es, anticiparse a la realidad futura en el presente, de acuerdo con análisis y estudios tecnocientíficos y socioeconómicos realizados en este momento.

Es en este pasaje que, en primer término, se puede apreciar el posicionamiento de una nueva figura social, como lo es la "ciudadanía del mundo", entendida esta como una condición reivindicatoria y deseable donde la extensión, la propiedad y la certidumbre dependen de la participación y la adherencia de las personas para preservar los derechos humanos, aun fuera de un determinado referente territorial, ya que estos les son inseparables, además de exigibles universalmente en todo momento. Es así como la "ciudadanía del mundo" se yergue como un concepto bajo el cual la justicia y el sentido de pertenencia se deben constituir como sus más relevantes características, tal como asevera Cortina (1997). En este orden de ideas, advierte Tuvila Rayo (2010, p. 5), sus rasgos se aprecian a través del

[...] reconocimiento de unos derechos y obligaciones ciudadanas (status legal), [en] la responsabilidad contraída en dicha relación (status moral) y el sentido de pertenencia al grupo desde el respeto a la diversidad cultural (identidad intercultural) en el seno de una sociedad abierta, compleja, plural y multicultural.

Retomando esta configuración social y colectiva que Cortina y Tuvila Rayo mencionan en sus posturas, esta nueva ciudadanía debe acoger y exhortar una praxis de la igualdad y la equidad, así como también el respeto por la dignidad humana y la naturaleza; igualmente, preservar lo distintivo y propio de cada colectivo social, y no apelar tan solo al discurso moral que ha prevalecido por años. Con ello, será posible romper la tendencia de pensar que basta con el discurso para llegar a ser un mejor ser humano. Cuando el discurso está más preocupado por el "decir" que por el "hacer", se disocian las palabras y se evidencia la falta de pertenencia y compromiso entre las personas. Cortina lo expresa categóricamente: "no puedo exigir como moral, como humano, un derecho que no esté dispuesta a exigir con igual fuerza para cualquier otro" (1997, p. 234), en tanto que Tuvila Rayo señala que lo distintivo de esta nueva ciudadanía es: 
[...] ese rasgo esencial que caracteriza a los miembros de una comunidad que favorecen la convivencia pacífica (justicia social), se comportan según unos valores acordados (derechos humanos) y participan activa y públicamente (ciudadanía activa y responsable) en la búsqueda de soluciones alternativas y posibles a las distintas problemáticas sociales. (2010, p. 4)

Tabla 2.

Pronunciamientos en las declaraciones universales sobre la calidad de vida de los seres humanos

Pronunciamiento
Principio 1
El hombre tiene derecho fundamental a la libertad, la
igualdad y el disfrute de condiciones de vida adecuadas,
en un medio ambiente de calidad tal que le permita llevar
una vida digna y gozar de bienestar, y tiene la solemne
obligación de proteger y mejorar el medio ambiente para
las generaciones presentes y futuras.

Declaración

Declaración sobre

el Medio Ambiente

Humano (Naciones

Unidas, Estocolmo,

1972)

Principio 8

El desarrollo económico y social es indispensable para asegurar un ambiente de vida y trabajo favorable y para crear las condiciones necesarias de mejora de la calidad de vida.

Principio 8
Para alcanzar el desarrollo sostenible y una mejor calidad de
vida para todas las personas, los Estados deberían reducir
y eliminar las modalidades de producción y consumo
insostenibles y fomentar políticas demográficas apropiadas.

Declaración de

Río sobre el Medio

Ambiente y el

Desarrollo (Naciones

Unidas, Río de

Janeiro, 1992

Punto 13: el medio ambiente mundial sigue deteriorándose. Continúa la pérdida de biodiversidad; siguen agotándose las poblaciones de peces; la desertificación avanza, cobrándose cada vez más tierras fértiles; se hacen evidentes los efectos Declaración sobre el Desarrollo Sostenible (Naciones Unidas, Johannesburgo, 2002) adversos del cambio del clima, los desastres naturales son más frecuentes y más devastadores, y los países en desarrollo se han vuelto más vulnerables, en tanto que la contaminación del aire, el agua y los mares sigue privando a millones de seres humanos de una vida digna.

Punto 8: reafirmamos también la importancia de la libertad, la paz y la seguridad, el respeto de todos los derechos humanos, entre ellos el derecho al desarrollo y el derecho a un nivel de vida adecuado, incluido el derecho a

Declaración sobre el Futuro que Queremos (Naciones Unidas, Río de Janeiro, 2012) la alimentación, el Estado de Derecho, la igualdad entre los géneros, el empoderamiento de las mujeres y el compromiso general de lograr sociedades justas y democráticas para el desarrollo.

Fuente: elaboración propia con base en las referencias citadas

Esta "ciudadanía del mundo" antes aludida, por ende, debe permitir que se posicione en todos los seres humanos, tanto individualmente como colectivamente, una serie de nuevas conductas 
y relaciones de responsabilidades, como también corresponsabilidades. Es ahí donde comienza una travesía, y también una reorganización de conductas más efectivas. Conductas que, primero, nos alejen de un antropocentrismo "fuerte", donde el ser humano se posiciona con una potestad y superioridad de carácter absoluto sobre su entorno, y donde no es factible, cuanto más imposible, pensar en una relación moral con su medio, y segundo, que nos ayuden a afirmarnos en uno más "sabio", de acuerdo con la situación del escenario y circunstancias del mundo, que fortalezca una nueva solidaridad que sea patente con la generación presente (solidaridad sincrónica) y también con las generaciones venideras (solidaridad diacrónica) (Norton, 1984; Tamames, 1984; Novo y Ángeles Murga, 2010). En otras palabras, como asevera imperativamente Hans Jonas, "Actúa de tal modo que los efectos de tu acción sean compatibles con la permanencia de una vida auténtica” (citado por de Siqueira, 2009, p. 172), que no sitúe en riesgo la permanencia y continuidad de la sociedad humana.

Por otra parte, esta nueva ciudadanía debe aspirar, como señala Yunus (1998), a redirigir la evaluación del progreso social y económico a partir de los que se encuentran en la escala social más baja, con el fin de encontrar modos de vida más frugales y así abatir el despilfarro y la dilapidación de bienes y recursos.

De acuerdo con Argandońa (2010, p. 2), la cualidad que distingue a esta actitud frugal en las personas, es que se

[...] desarrolla voluntariamente un estilo de vida sencillo: no crea necesidades, no posee más de lo necesario, renuncia a un nivel de vida superior, se rodea de pocos bienes y éstos son poco lujosos. Esto puede ser compatible con un elevado nivel de riqueza, pero no materializada en bienes que facilitan servicios de consumo, incluidos los de consumo suntuario: el número, tamańo y calidad de la vivienda y de los medios de transporte, los muebles, ropa, etc., que posea.

A esta nueva construcción teórica de "ciudadanía del mundo frugal", habría que añadir el vocablo "segura", que involucraría la satisfacción y cobertura de los "costos del hombre" que menciona Perroux, los cuales están relacionados con los aspectos de cobertura de salud, alimentación adecuada, acceso a la educación y cultura (Guillén Romo, 2008), pero además acogería al concepto de seguridad humana desplegado por el Programa de las Naciones Unidas para el Desarrollo (pNud) (1993, p. 2), en el Informe sobre Desarrollo Humano de 1993, donde declara:

El concepto de seguridad humana debe cambiar, evolucionando de tal manera que de basarse exclusivamente en la seguridad nacional pase a destacar mucho más la seguridad de la gente, de la seguridad mediante el armamentismo hacia la seguridad mediante desarrollo humano, de la seguridad territorial a la seguridad alimentaria, en el empleo y el medio ambiente.

La seguridad humana, como señala Fernández Pereira (2005, p. 11), "hace hincapié en la necesidad de tomar medidas preventivas para disminuir la vulnerabilidad y reducir al mínimo los riesgos para los derechos, la seguridad y la vida de la gente". Así, involucra aspectos tan diversos como la seguridad ambiental, la seguridad alimentaria, la seguridad económica, la seguridad en salud, la seguridad política, la seguridad comunitaria y la seguridad de orden personal (Villanueva Ayón, 2000; Mack, 2005; Ariza, 2010). En consecuencia, promueve las garantías de salvaguardar las libertades individuales, así como las libertades colectivas, considerando que es inherente a todos los seres humanos y conlleva el respeto de todo lo esencial para la existencia y el sostenimiento de la humanidad.

\section{Conclusiones}

Es importante reconocer que las políticas económicas neoliberales nos han conducido a un sistema donde las desigualdades sociales han aumentado. Estas desigualdades implican pobreza, desempleo y enfermedad, entre otras desventajas que menguan la calidad de vida y la sustentabilidad de los sistemas 
en que subsistimos. La modernización no ha sido un proceso integral y practicable, sino truncado, fragmentario y perturbador, con muy serios desequilibrios que han ensombrecido el panorama y cuyos síntomas se han manifestado en una desarticulación social de la eufemísticamente llamada "modernidad". Esta modernidad ha hecho desaparecer el sentido de lo comunitario y se ha evidenciado en las dificultades para lograr una afinidad social, y también ha conllevado un cambio fundamental en la forma de convivencia.

Por lo tanto, el término de desarrollo sustentable cobra gran importancia, considerando la perpetuación del medio natural y físico, pero además busca garantizar las oportunidades futuras de satisfacción de la gente, a través de un cambio en la calidad de vida, particularmente hacia lo moderado y seguro, donde el replanteamiento del que se habla requiere superar las barreras de la mentalidad economicista del sistema cultural actual, para articular los aspectos ambientales con los aspectos sociales. Esto significa que los "sectores y países ricos deben necesariamente reducir hoy sus niveles de vida a fin de que sus consumos actuales no hipotequen el presente y el futuro” (Gutiérrez Garza y González Gaudiano, 2010, p. 131).

La anterior reflexión nos conduce a observar en esta nueva edificación teorizante, de una "ciudadanía del mundo frugal y segura”, el elemento para cuidar el carácter universal de la vida: la dignidad humana, y reconocer el carácter complementario de los distintos ámbitos que tienen que ver con la humanidad, como lo son las esferas socioeconómica, ambiental y política, para así incrementar la gama de oportunidades de los seres humanos, con el fin de arribar a una mejor calidad de vida. Esta "ciudadanía del mundo frugal y segura", se constituiría, aludiendo a Godard, "en un principio de no dictadura intergeneracional: ni dictadura del presente, que se manifestaría en una indiferencia por el futuro, incluso el lejano, ni dictadura del futuro que impondría el sacrificio de las generaciones actuales" (Gutiérrez Garza y González Gaudiano, 2010, p. 131). Sin embargo, para responder a ello, en los tiempos actuales de globalización, es conveniente fortalecer cuatro elementos que son imprescindibles en la sociedad humana: el ostentar una adecuada cohesión social, exhibir una buena calidad en los liderazgos, tener estabilidad institucional, con independencia de las tendencias ideológicas y, finalmente, tener ideas asentadas en el interés, no solamente nacional, sino además en lo global (Conill, 2009; Ferrer, 2010).

Es en tal derrotero que se gozaría de una vida con decoro y capacidad de elección, que produciría un ser humano con una vida plena, sustentada en la integración de valores, derechos y capacidades, vinculados a los demás por lazos de reconocimiento e interés recíproco, donde lo fundamental es lo correlativo a la vida en común, que privilegiaría lo colectivo sobre lo individual, pero que a la vez consentiría un trato similar que permitiría a todo ser humano plasmar y realizar por igual su particular plan de vida, desde los ámbitos del seno familiar, comunitario y desde las complejas y diversas relaciones interpersonales. Por último, este nuevo constructo, "ciudadanía del mundo frugal y segura", sintetiza los criterios contenidos en las definiciones de calidad de vida y desarrollo sustentable. 


\section{Referencias}

Ardila, R. (2003). Calidad de vida: una definición integradora. Revista Latinoamericana de Psicología, 35(2), 161-164.

Argandońa, A. (2010). Frugalidad. Barcelona/Madrid: IESE Business School-Universidad de Navarra, Documento de Investigación DI-873.

Arita-Watanabe, B. Y. (2005). La capacidad y el bienestar como dimensiones de estudio de la calidad de vida. Revista Colombiana de Psicología, 14, 73-79.

Ariza, N. (2010). La aplicabilidad del concepto de seguridad humana en América Latina y el Caribe: El desarrollo humano como fuente de seguridad. OASIS, 15, 33-51.

Bustelo-Graffigna, E. (2008). ¿Vida o calidad de vida? Salud Colectiva, 4(2), 143-147.

Cantú-Martínez, P. C. (2008). Desarrollo sustentable. Conceptos y reflexiones. México: Ed. Universidad Autónoma de Nuevo León.

Cantú-Martínez, P. C. (2012). El Axioma del Desarrollo Sustentable. Revista de Ciencias Sociales, 137(3), 83-91.

Cantú-Martínez, P. C. (2013a). Hacia la edificación de una sociedad más sustentable. Ciencia UANL, 16(63), 45-53.

Cantú-Martínez, P. C. (2013b). Conciencia y construcción social de la sustentabilidad. Ciencia UANL, 16(62), 36-44.

Cantú-Martínez, P. C. (2013c). Razonamiento socioambiental acerca del desarrollo sustentable. Ciencia UANL, $16(64), 32-36$

Comisión Mundial sobre el Medio Ambiente y el Desarrollo (1988). Nuestro Futuro Común. Bogotá: Alianza Editorial Colombiana.

Conill, J. (2009). Ciudadanía económica en la jungla global. En N. Guzmán (Comp.), Sociedad, desarrollo y ciudadanía en México (pp. 273-288). México: Limusa.

Cortina, A. (1997). Ciudadanos del mundo. Hacia una teoría de la ciudadanía. Madrid: Alianza Editorial.

De Siqueira, J. E. (2009). El principio de responsabilidad de Hans Jonas. Revista Bioethikos, 3(2), 171-193.

Elizalde-Hevia, A. (2003). Desde el desarrollo sustentable hacia sociedades sustentables. En B. Blejmar (Comp.), Liderazgo y desarrollo sustentable (pp. 41-76). Buenos Aires: Ed. Manantial.

Fernández-Pereira, J. P. (2005). Seguridad humana (tesis inédita de Doctorado). Universidad Autónoma de Barcelona, Barcelona, España.

Ferrer, A. (2010). Raúl Prebisch y el dilema del desarrollo en el mundo global. Revista Cepal, 101, 7-15.

Filmus, D. (1996). Estado, sociedad y educación. Proceso y desafíos. Buenos Aires: Editorial Troquel.

Flórez, M. (2002). Apuntes para una ética desde el movimiento social ambiental y las organizaciones ciudadanas. En E. Leff (Coord.), Ética, vida, sustentabilidad (pp. 199-208). México: Pnud / Red de Formación Ambiental para América Latina y el Caribe.

García-Viniegras, C. R. V. (2005). El bienestar psicológico: Dimensión subjetiva de la calidad de vida. Revista Electrónica de Psicología Iztacala, 8(2), 1-20.

Guillén-Romo, H. (2008). François Perroux: Pionero olvidado de la economía del desarrollo. Mundo Siglo XXI, 11, 11-22. Gutiérrez-Garza, E. y González-Gaudiano, E. (2010). De las teorías del desarrollo al desarrollo sustentable. México: Ed. Siglo XXI.

Hernández, A. G. (2008). El desarrollo humano. En A. G. Hernández (Comp.), El desarrollo como problema. ¿Igualdad de qué? (pp. 35-48). Caracas: Universidad Central de Venezuela/Consejo de Desarrollo Científico y Humanidades. 
Hernández-Vela Salgado, E. (2001). La cultura como fundamento de la paz y el desarrollo. En I. Cid (Comp.), Diversidad cultural, economía y política en un mundo global (pp. 77-84). México: unam-Facultad de Ciencias Políticas y Sociales.

Herrera, A. O., Scolnik, H. D., Chichilnisky, G., Gallopin, G. C., Hardoy, J.E., Mosovich, D. ... Talavera, L. (2004). ¿Catástrofe o Nueva Sociedad? Modelo Mundial Latinoamericano 30 años después. Buenos Aires: Centro Internacional de Investigaciones para el Desarrollo/IIED-América Latina.

Jacobs, M. (1996). La economía verde. Medio Ambiente, desarrollo sostenible y la política del futuro. Barcelona: Icaria/Fuhem. López-Vizcaíno, M. E. y Sánchez-Fernández, P. (2009). La medición de la calidad de vida en las comarcas gallegas. Revista Galega de Economía, 18(1), 1-20.

Lora, E. (Coord.) (2008). Calidad de vida: Más allá de los hechos. México: Banco Interamericano de Desarrollo / Fondo de Cultura Económica.

Macía-Arce, X. C. (2009). Consideraciones teóricas sobre el concepto calidad de vida en la sociedad de la información. Revista Electrónica Teoría de la Educación, 10(2), 246-262.

Mack, A. (2005). El concepto de seguridad humana. Papeles, 90, 11-18.

Naciones Unidas (1992). Declaration of the United Nations Conference on the Human Environment. Recuperado el 9 mayo del 2014, de http://www.unep.org/Documents.Multilingual/Default.asp?DocumentID=97 \&ArticleID $=1503 \& \mathrm{l}=\mathrm{en}$.

Naciones Unidas (1992). Declaración de Río sobre el Medio Ambiente y el Desarrollo. Recuperado el 6 de mayo del 2014, de http://www.un.org/spanish/esa/sustdev/agenda21/riodeclaration.htm.

Naciones Unidas (2002). Informe de la Cumbre Mundial sobre el Desarrollo Sostenible. A/CONF.199/20. Johannesburgo: Naciones Unidas.

Naciones Unidas (2012). El futuro que queremos. A/CONF.216/L.1. Río de Janeiro: Naciones Unidas.

Nava-Galán, M. G. (2012). La calidad de vida: Análisis multidimensional. Enfermería Neurológica 11(3), 129-137.

Norton, B. G. (1984). Environmental Ethics and Weak Anthropocentrism. Environmental Ethics, 6(2), 131-148.

Novo, M. y Angeles-Murga, M. (2010). Educación ambiental y ciudadanía planetaria. Revista Eureka sobre Enseñanza y Divulgación de la Ciencia, 7 (número extraordinario), 179-186.

Peña-Guerrero, R. (2001). Ética y relaciones internacionales: Reflexiones básicas. En I. Cid (Comp.), Diversidad cultural, economía y politica en un mundo global (pp. 41-47). México: unAM-Facultad de Ciencias Políticas y Sociales.

Programa de las Naciones Unidas para el Desarrollo (1993). Informe sobre desarrollo humano 1993. Espańa: PNUDCideal.

Robaina, H., Fernández, A. T. y Ramírez, A. R. (2011). Calidad de vida: Algo más que un concepto. MediSur, 9(5), 3-6.

Rodríguez, N. y García, M. (2005). La noción de calidad de vida desde diversas perspectivas. Revista de Investigación, 57, 49-68.

Rull, V. (2010). El mito del desarrollo sostenible. Collectanea Botanica, 29, 103-109.

Salas-Zapata, C. y Grisales-Romero, H. (2010). Calidad de vida y factores asociados en mujeres con cáncer de mama en Antioquia, Colombia. Revista Panamericana de Salud Pública, 8(1), 9-18.

Sánchez-de Madariaga, I. (2004). Infraestructuras para la vida cotidiana y calidad de vida. Ciudades, 8, 101-133

Sierra-Fonseca, R. (2001). Integración social y equidad en la perspectiva del desarrollo humano. Tegucigalpa: PNUD.

Southcliffe-Ashton, A. (2008). La Revolución industrial, 1760-1830. México: Fondo de Cultura Económica. 
Tamames, R. (1984). Utopia y contrautopía. Barcelona: Plaza y Janés.

Tuvilla-Rayo, J. (2010). La visión holistica e integrada de la formación ciudadana en los centros educativos. Consejería de Educación. Memorias del I Congreso de Inspección de Andalucía: Competencias básicas y modelos de intervención en el aula (pp. 1-20). Málaga: Junta de Andalucía.

Unión Internacional para la Conservación de la Naturaleza (UICN) (2008). Transición a la sostenibilidad: Hacia un mundo humanitario y diverso. Gland: UICN.

Urzúa, A. y Caqueo-Urizar, A. (2012). Calidad de vida: Una revisión teórica del concepto. Terapia Psicológica, 30(1), 61-71.

Villanueva-Ayón, M. (2000). Seguridad humana ¿Una ampliación del concepto de seguridad global? Revista Mexicana de Politica Exterior, 59, 106-130.

Xercavins, J., Cayuela, D., Cervantes, G. y Sabater, A. (2005). Desarrollo sostenible. Barcelona: Ed. UpC.

Yunus, M. (2003). Hacia un mundo sin pobreza. Santiago de Chile: Editorial Andrés Bello. 
